\title{
PARTICLE-JET INTERACTIONS IN AN MHD SECOND STAGE COMBUSTOR
}

$\mathrm{AN} / \mathrm{CP}-75446$

DE92 016390

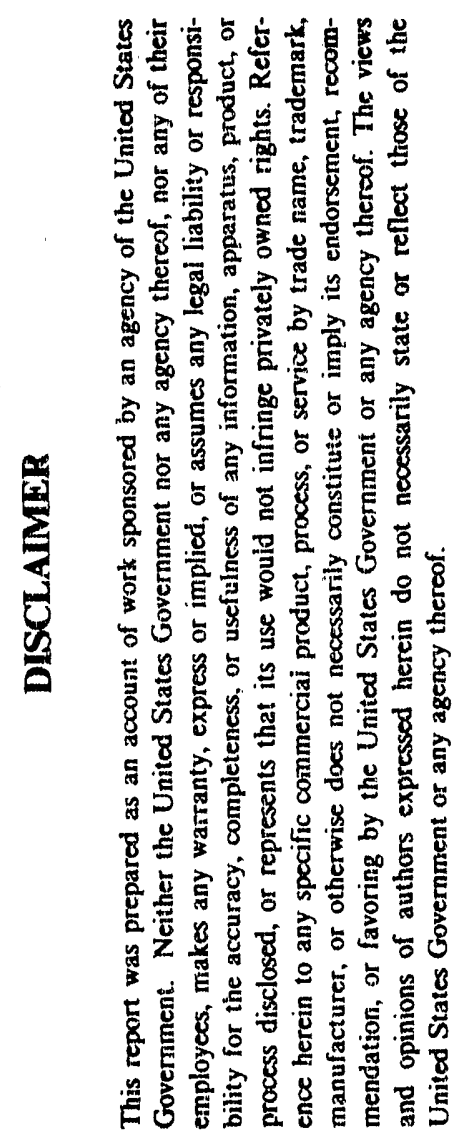

\author{
S.A. Lottes and S.L. Chang \\ Energy Systems Division \\ Argonne National Laboratory \\ 9700 South Cass Avenue \\ Argonne, Illinois 60439
}

manuscript submitted to

The 30th Symposium of Engineering Aspects of Magnetohydrodynamics

June 29-July 2, 1992

Baltimore, Maryland

\section{MASTER}




\title{
PARTICLE-JET INTERACTIONS IN AN MHD SECOND STAGE COMBUSTOR
}

\author{
S.A. Lottes and S.L. Chang \\ Argonne National Laboratory \\ 9700 South Cass Avenue \\ Argonne, IIIInols 60439
}

\begin{abstract}
An Argonne two-phase combustion flo: viniputer code is used to simulate rearting flows to aid in the development of an advanced combustor for magnetohydrodynamic power generation. The combustion code is a general hydrodynamics computer code for two-phase, two-dimensional, steady state, rurbulent, and reacting flows, based on mass, momentum, and energy conservation laws for multiple gas species and particles. The combustion code includes turbulence, integral combustion, and particle evaporation submodels. A recently developed integral combustion submodel makes calculations more efficient and more stable while still preserving the major physical effects of the complex combustion processes. The combustor under investigation is a magnetohydrodynamic second stage combustor in which opposed jets of oxidizer are injected into a confined cross-stream of hot coal gas flow following a first stage swirl combustor. The simulation is intended to enhance the understanding of seed particle evaporation in the combustor and evaluate the effects of combustor operating conditions on seed particle evaporation and vapor dispersion, which directly affect overall magnetohydrodynamic power generation. Computation results show that oxidizer jet angle and particle size may greatly affect particle evaporation and vapor dispersion. At a jet angle about 130 degrees, particle evaporation rate is the highest because of the highest average gas temperature. As particle size increases beyond 10 mictons in diameter, ths effects of particle size on wall deposition rate, evaportaion deli,y, and downstream seed vapor dispersion become more pronounced.
\end{abstract}

\section{NOMENCLATURE}

B Evaporation transfer number

CL Convergence level

$\mathrm{C}_{d} \quad$ Farticle drag coefficient

Cp specific heat $(\mathrm{J} / \mathrm{mol} / \mathrm{K})$

D Combustor hydraulic diameter (m)

$\mathrm{H}_{v}$ Latern heat $(\mathrm{J} / \mathrm{kg})$

h Enthalpy $(J / \mathrm{kg})$

k Turbulence intensity $(\mathrm{J} / \mathrm{kg})$

K Gas thermal conductivity (W/mK)

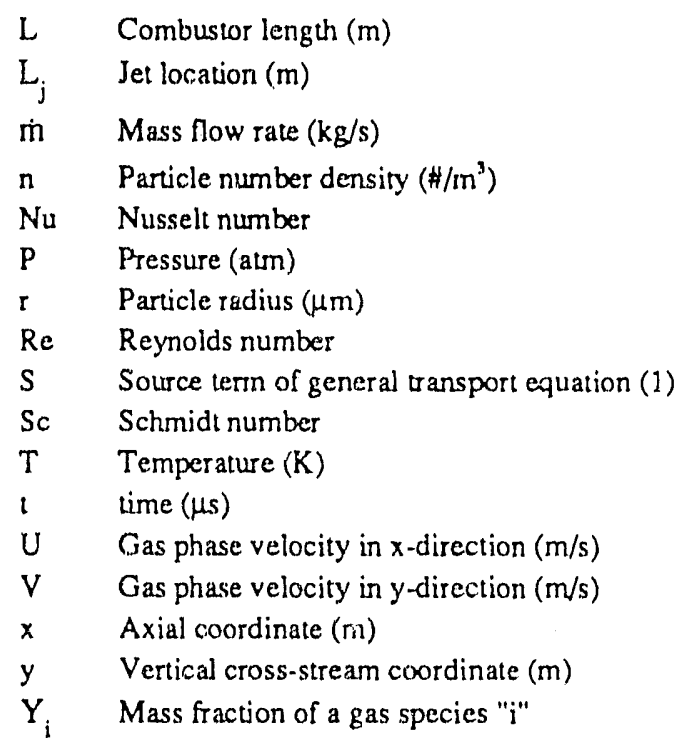

\section{Greek Letters}

$\begin{array}{ll}\varepsilon & \text { Turbulence dissipation }(\mathrm{J} / \mathrm{kg} \cdot \mathrm{s}) \\ \Gamma & \text { Diffusion coefficient } \\ \mu & \text { Viscosity }(\mathrm{N}-\mathrm{s}) \\ \phi & \text { Stoichiometric mass ratio of oxidizer and fucl } \\ \rho & \text { Density }\left(\mathrm{kg} / \mathrm{m}^{3}\right) \\ \tau & \text { Richness } \\ \theta & \text { Gas volume fraction } \\ \xi & \text { General flow variable } \\ \zeta & \text { Extent of reaction }\end{array}$

\section{Subscripts}

$\begin{array}{ll}\text { o } & \text { Reference } \\ \text { b } & \text { Boiling } \\ \text { fu } & \text { Fuel species } \\ \text { ir } & \text { Inert species } \\ \text { pr } & \text { Product species } \\ \text { ox } & \text { Oxidizer species } \\ \text { res } & \text { Residual quartity } \\ \text { s } & \text { Solid phase variable } \\ \text { sv } & \text { Seed vapor species }\end{array}$


Turbulence

Axial direction

Cross-stream direction

\section{INTRODUCTION}

The concept of a magnetohydrodynanic (MHD) power plank, which depends upon the interaction between magnetic fields and an electrically conducting fluid flow to generate electrical power, has attracted much interest in the utility industry because it can attain higher overall efficiency and produce less pollutants compared to a conventional coal-fired power plant [1-2]. The U.S. Department of Energy has been sponsoring a national program for the development of a proofof-concept MHD power plant in which industries, universities, and national laboratories have participated. Under this program, Argonne National Laboratory (ANL) provides technical support to the industries who are building the combustor, MHD channel, and other facilities. One major activity at ANL is the use of computer simulation to aid in the design of a $50 \mathrm{MWt}$ MHD combustor at TRW [3-6]. The combustor is a two-stage pulverized coal combustor upstream of the MHD generator [7]. The first stage is a swirl combustor operated under substoichiometric conditions to minimize NOx formation while most of slag is removed. A second stage combustor follows the first stage combustor. in the second stage combustor, seed material, i.e., potassium, and additional oxidizer are injected to oblain desired plasma stoichiometry and temperature for successful MHD channel operations.

Among other important issues regarding the operation of a second stage MHD combustor, seed particle evaporation, seed vapor dispersion, and combustion are studied to determine the effects of combustor operating conditions on MHD channel performance. One of the major concerns is the distortion of gas temperature and seed vapor profiles, caused by incomplete mixing which may significantly lower the electric conductivity of the gas and subsequently the MHD channel performance. Computer simulation is an efficient and cost-effective tool to help design an advanced combustor because it provides indepth information about the two-phase combustion flow and the flexibility to experiment with a wide range of operating conditions at relatively low cost.

Some concems regarding the simulation of a two phase combustion flow include computational speed of available computers and numerical stability of the employed computer codes. Recent advances in high-speed supercomputers, computational techniques to solve the coupled partial differential equations of a qurbulent flow [8-9], and combustion related modeling, e.g., turbulence, combustion, and jet mixing rnudels [10.11], have encouraged people to develop conprehensive computer models to simulate the complex processes of nuid mixing and reaction in a combustion system.
A team of ANL and University of Mlinois at Chicago developed a comprehensive combustion flow computer code. The code was originally written for air-breathing propulsion engines [12] and has been modified for various applications in coal-[ured combustors [13] and internal combustion engines [14]. For the present study, the code has been modified extensively over the past few years at ANL. The study investigates important issues related to the performance of an MHD second stage combustor. The issues include particle evaporation, vapor dispersion, gas ionization, combustion, jet penetration, and fluid mixing. This paper focuses on seed particle evapuration and vapor dispersion in the combustion flow.

\section{SIMULATION APPROACHES}

An ANL two-phase two-dimensional combustion computer code was used to simulate combustion flow patterns in an MHD second stage combustor. The code solves transport equations for five gaseous species (fuel, oxidizer, product, inert, and seed) and solid particles over a range of sizes. General conservation laws, expressed by elliptic-type partial differential equations, are used in conjunction with rate equations goveming the mass, momentum, enthalpy, species, turbulent kinetic energy, and turbulent dissipation for a twophase reacting flow. The associated submodels of this code inciude an integral combustion, a two-parameter turbulence, a particle evaporation, and other interfacial submodels. A recently developed integral combustion submodel replacing an Arrhenius type differential reaction submodel has been implemented to improve numerical convergence and enhance numerical stability. A two-parameter turbulence submodel is modified for both gas and solid phases. The evaporation submode! treats not only particle evaporation but size dispersion. Interfacial submodels use simple correlations to model interfacial momentum and energy transfer.

\section{General Transport Equations}

For convenience of numerical formulation the governing usansport equations for both gas and solid phases are put in a common form:

$$
\frac{\partial J}{\partial x}+\frac{\partial J}{\partial y}=S_{s}
$$

in $w^{2}$.ich $\xi$ is a general flow variable, $S$ is a source (or sink) term, and J's as combined convective and diffusive nux terms.

Gas species are assumed to be a perfect gas mixture. The convective and diffusive flux terms of a gas Mow variable can be written as. 


$$
\begin{aligned}
& J_{x}=\theta \rho U \xi-\Gamma_{\xi} \frac{\partial \xi}{\partial x} \\
& \text { for } \xi=1, U, V, Y_{N^{\prime}} Y_{i r} Y_{w^{\prime}}, \tau, h, k_{,} \text {or } \varepsilon \\
& J_{y}=\theta \rho V \xi-\Gamma_{\xi} \frac{\partial \xi}{\partial y} \\
& \tau=\frac{\phi Y_{N}-Y_{o x}+Y_{0}}{\phi+Y_{0}} \\
& Y_{m}=1-Y_{M}-Y_{a}-Y_{i r}-Y_{r v}
\end{aligned}
$$

where, $\theta$ is gas volume fraction, $p$ is density, $\Gamma$ is effective diffusion coefficient, $\phi$ is the stoichiometric mass ratio of oxidizer and fuel, and $Y_{0}$ is a reference concentration. $U$ and $V$ are velocity components, $Y_{\Gamma} Y_{t r} Y_{v v}$ are mass fractions of gaseous species of fuel, inert, and seed vapor, respectively, $\tau$ is richness, $h$ is enthalpy, $k$ is turbulent kinetic energy, and $\varepsilon$ is turbulent dissipation rate. Note that concentrations of oxidizer and product species are not solved directly from a transport equation. Since a transport equation with no source term is more stable in the solution routines, the transport equation for oxidizer concentration is replaced by a richness equation which eliminates the reaction sink term if richness is defined in equation (3). As soon as the richness and fuel species transport equations are solved, oxidizer concentration can be easily obtained from equation (3). After all the other species are solved, product concentration can be obtained from the species conservation equation (4).

The solid phase equations include those governing number ciensity $(\mathrm{n})$, velocities (momentum) $\left(\mathrm{U}_{1}, \mathrm{~V}_{\mathrm{R}}\right)$, and temperature $\left(T_{0}\right)$. For each particle size group, the convective and diffusive flux terms of number density are:

$$
\begin{aligned}
& J_{x}=n U_{1}-\Gamma_{0} \frac{\partial n}{\partial x} \\
& J_{y}=n V_{1}-\Gamma_{0} \frac{\partial n}{\partial y}
\end{aligned}
$$

and the flux terms for particle momentum and temperature are:

$$
\begin{gathered}
J_{x}=n U_{.} \xi \\
J_{y}=n V_{0} \xi \\
\text { for } \xi=U_{A}, V_{,} \text {or } T_{.} .
\end{gathered}
$$

The source term of particle nurnber density accounts for particle evaporation, while the diffusion term accounts for particle dispersion due to interaction with the rurbulence of the gas phase. The interactions between phases are all included in the source terms. For exarnple, a momentum sink in gas flow accounting for particle drag effects is also a momentum source for the solid flow.

\section{Assoclated Submodels and Empirical Correlations}

To determine source terms and effective diffusion coefficients of the transport equations for both gas and solid phase flow variables, submodels and empirical correlations are needed. For the source terms of the transport equations of fuel concentration and enthalpy, a submodel is required to determine fuel consumption and heat release rates. For seed vapor concentration and particle number density, a submodel is required to determine particle evaporation and size dispersion rates. For gas and solid velocities, conelations of interfacial drag force are required to determine mornentum exchanged between phases. For gas enthalpy and solid temperature, correlations of interfacial heat transfer are required to determine energy exchange between phases. For the effective diffusion coefficients of all transport equations, a submodel is required to determine turbulent diffusivity for both gas and solid phases.

An integral one-step combustion submoriel has been developed to make numerical calculation of the compli:x combustion processes in the present study faster and more efficient while still preserving the major physical effects of the combustion on the flow development. The model replaces a previous differential combustion model which used an Arrhenius type rate equation. The new model is found to substantially enhance the numerical stability of reacting flow computations with the computer code. The model assumes that the overall reaction progress and its physical effects can be expressed by empirical correlations or tabulated data relating the extent of reaction (or the fraction of fuel consumed) and the accumulated heat of combustion to a flow time scale instead of a reaction lime scale. Detailed kinetics calculations provide data to tabulate or correlate an extent of reaction as a function of time. For this study, detailed kinetics calculations are carried out using NASA's General Chemical Kinetics Program [15]. For a combustion process at a reaction pressure of $5.7 \mathrm{~atm}$ and initial temperature of $1750 \mathrm{~K}$, a correlation of the extent of reaction $\zeta$ can be written as,

$$
\begin{gathered}
\zeta=0.1495 \ln (80 t+1) \\
\text { for } t \leq 0.1 \mu \mathrm{s} \\
\zeta=1-\exp \left(-0.705 t^{0.25}\right) \\
\text { for } 0.1<1 \leq 0.25 \mu \mathrm{s} \\
\zeta=0.57-0.1774 \exp \left(-0.78(1-0.25)^{\mathrm{iot}}\right) \\
\text { for } t>0.25 \mu \mathrm{s}
\end{gathered}
$$

A similar correlation for heat of combustion is also 
obtained from the plotted results. These relations are incorporated into the source terms of the gas enthalpy and fuel concentration transport equations. More details of the integral combustion submodel were reported at the 1991 SEAM Conference [4].

A tw: parameter turbulence submodel is used to simulate the turbulent rarsport of gas and solid phases. Based on the work of Launder and Spalding [8], turbulent viscosity $\mu_{1}$ is defined as:

$$
\mu_{1}=0.09 \rho \frac{k^{2}}{\varepsilon}
$$

in which the two turbulent parameters $k$ and $\varepsilon$ are solved by two turbulent transport equations. For gas momentum equations, an effective viscosity (or diffusion coefficient) is defined as the sum of gas viscosity and turbulent viscosity. For equations of other gas flow variables, effective diffusivities are assumed to be proportional to the effective viscosity. Similarly, diffusion coefficients for particle number density are assumed to be related to gas turbulent viscosity and account for the effect of particle dispersion by gas iurbulence as a function of paritcle size. The larger the particle, the smaller the diffusiviry. The empirical formula for the particle diffusion coefficient used in this computer code is based on the work of Ward et al. [16].

$$
\Gamma_{\mathrm{p}}=\frac{\mu_{1}}{0.9 \rho\left(1+2 r+0.06 r^{2}\right)}
$$

where $r$ is particle radius (in $\mu \mathrm{m}$ ).

Treating a spray of particles in the Eulcrian framework, the number density is taken to be a function not only of the coordinates $x$ and $y$ but also of the particle radius. Thou and Chiu [10] used a simple mathematical function used to represent the inlet size distribution of a spray. A similar size distribution formula is used to represent the inlet seed particle flow. The formula is:

$$
\frac{d n}{d r}=a r^{4} \exp \left(-b r^{4}\right)
$$

where $a$ and $b$ are constants to be determined from total particle number density and mean particle radius.

A transport equation for the number density of a particle size group is derived by doing a balance over a $(x, y, r)$ space. Similar to the discretization of the $x$ and $y$ coordinate to yield the physical space computational grid, the particle size coordinate, $r$, is discretized to yield a set of particle size groups. The number density transport equation accounts for not only the fluxes in the physical coordinates but the rate of shift of the particle size spectrum due to particle evaporation, which causes evaporating particles to move from larget to smaller size groups. When particles evaporate, particle size decreases, seed vapor is added to the gas phase, momentum is added to the gas momentum, and latent heat is subtracted from the gas enthalpy. A correlation is used to predict particle evaporation rate in a convective field.

$$
\begin{aligned}
& \qquad \mathrm{m}=4 \pi \mathrm{r}(\mathrm{K} / \mathrm{Cp}) \mathrm{Nu}_{\mathrm{c}} \ln (1+\mathrm{B}) \\
& \text { where } \\
& \text { and } \mathrm{Nu}_{\mathrm{c}}=1+.276 \mathrm{Rc}^{3} \mathrm{Sc}^{3 \mathrm{3}} \\
& \qquad \mathrm{B}=\frac{\mathrm{Cp}\left(\mathrm{T}-\mathrm{T}_{\mathrm{D}}\right)}{\mathrm{H}_{\mathrm{v}}}
\end{aligned}
$$

In the above equations, $\mathrm{X}$ is gas thermal conductivity, $\mathrm{Cp}$ is gas sperific heat, and $\mathrm{Nu}, \mathrm{Re}, \mathrm{Sc}$, and $\mathrm{B}$ are Nusselt, Reynolds, Schmidh and evaporation transfer numbers respectively. Reynolds number is defined using slip velocity.

Gas thermal conductivity increases with temperature and decreases with pressure. In the temperarure range of this study, from 300 to $3000 \mathrm{~K}$, gas thermal conductivity varies more than an order of magnitude and pressure dependency becomes apparent at high temperature (above $2000 \mathrm{~K}$ ). In a two phase flow variation of thermal conductivity of the gas with temperature has a large effect on the rate of heat exchange between the phases. By using the published data, an empirical correlation of gas thermal conductivity has been established including both temperature and pressure $(\mathrm{P})$ dependency. The correlation is written as,

$$
\begin{gathered}
\mathrm{K}=0.0223+0.0977(\mathrm{~T}-250) / 1550 \\
\text { for } \mathrm{T}<1800 \mathrm{~K} \\
\mathrm{~K}=1+0.0977(\mathrm{~T}-1800) / 1550+ \\
(0.2904-0.182(\mathrm{P}-1) / 9)((\mathrm{T}-1800) / 1200)^{2.99} \\
\text { for } \mathrm{T}>1800 \mathrm{~K}
\end{gathered}
$$

In a two-phase flow, the solid flow is driven by the gas flow via the shear force generated between phases which depends on the velocity differential between phases. The larger the velocity differential, the bigger the interfacial force. The empirical formula for the particle drag coefficient used in the combustion code is,

$$
C_{d}=(24 / R e)\left(1+.15 R^{6 * 7}\right) /(1+B)
$$

Similarly, the empirical formula for paricie heat transfer coefficient is written in a correlation giving the Nusselt number. 


$$
\mathrm{Nu}=2+.654 \mathrm{Re}^{5} \mathrm{Sc}^{33}
$$

The momentum transfer rate between phases needed for the source terms of gas and particle mornentum transport equations is calculated based on the drag coefficient and a slip velocity. The convective heat $L$ iansfer rate between phases needed for the source terms of gas enthalpy and particle temperature transport equations is calculated based on the Nusselt coefficient and a slip temperature (temperature difference between gas and solid phases).

\section{Numerical Scheme}

Figure 1 shows the combustor under the investigation, an idealized rectangular box consisting of four solid side walls (front, back, top and bottom), an inlet for gas and particle flow (left), oxidizer injecion slots on both top and bottom walls representing distributed injection holes for the two-dimensional computation, and the exit (right). A two-dimensional computational domain is defined in a cross-sectional area in the middle of the combustor away from the viscous effects near the front and back walls as shown in Figure 1. The two. dimensional grid point system uses a horizontal $x$-axis and a vertical $y$-axis, and has the origin at the lower left comer. Evenly spaced grid points are used for the $y$-axis and variably spaced grid points are defined for the $x$-axis depending on the jet location. Dense grid points are selected near the jet opening where large flow property gradients are expected.

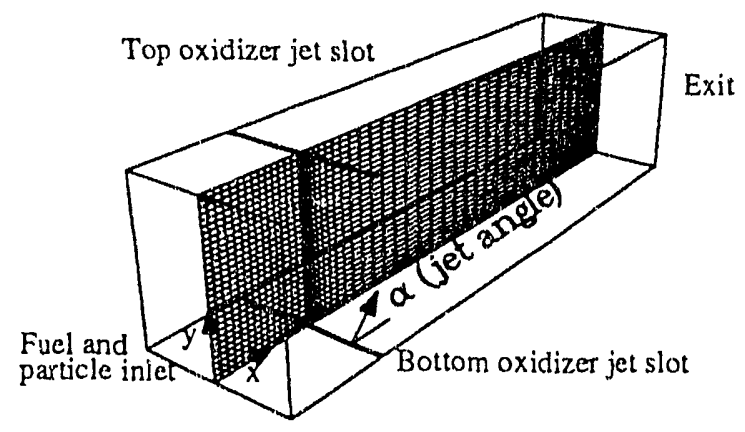

Figure 1 Combustor geometry and a two-dimensional computational grid with 54 by 32 nodes

A convergence and grid sensitivity study was conducted to identify convergence levels for both gas and particle phases in which computed variables have converged to four or more decimal digits over nearly all of the grid points, to identify a level of grid refinement in which computed variables change little upon further grid refinement and to identify a number of particle size groups (the discretization parameter for the particle phase) in which computed particle variables change little upon further refinement of particle size space. Based on results of the sensitivity study, a grid of 54 by 32 nodes was selected to represent the combustor configuration shown in Figure 1 and five particle size groups were used to represent the size distribution for two-phase combustion flow calculations. Acceptible convergence was judged to be achieved when the mass residual over all computational cells was reducet to less than $10^{-9}$ for the gas phase and $10^{-5}$ for the particle phase.

Most flow variables are assigned values at the inlet plane and jet openings in the side walls. A reference pressure is assigned at the midpoint of the inlet plane. Patankar's locally one way flow assumption [9] is applied to the outflow boundary, eliminating the need to specify the values of flow variables at the outflow boundary. In this formulation, the streamwise diffusion coefficients are taken to be zero at the outflow boundary. The side walls are impermeable for gas. Solids are allowed to deposit on the walls. A momentum wall function is used to bridge the near wall boundary layer. A staggered grid system was used for the numerical calculation, with the gas velocity components stored on the cell surfaces and all other physical quantities stored at the nodal points of each cell (or scalar cell). The governing partial differential equations are transformed into algebraic equations by integrating over the computational cell. These algebraic equations are solved using a line-by-line sweep in the primary flow direction to avoid numerical asymmetry.

A procedure added to the computer code dynamically alters the computational grid to adjust the area of the jet and maintain a specified jet mass flow rate constant during iteration woward the solution. The width of the jet slots is adjustable during computation so the total jet mass flow rate and jet velocity are fixed as defined by input values. When the jet velocity is specified at the jet inlet, the compressibility of jet inlet conditions affects the mass conservation of jet flow because pressure is no longer a free boundary condition, but rather needs to be determined from the flow solution in the interior.

\section{RESULTS AND DISCUSSION}

A parametric study has been performed to study particlejet interactions in reacting flows, and to investigate the effect of seed injection conditions on wall deposition and yapor dispersion. The study includes two parts of simulations: one assumes particles do not vaporize and the other assumes particles vaporize at a fixed temperature with a combined heat of fusion and vaporization. Common flow conditions used for the study are summarized in Table I. 


\begin{tabular}{|lr|}
\hline \multicolumn{2}{|c|}{ Table I Common Flow Conditions } \\
\hline Combustor Aspect Ratio (L/D) $=$ & 3.84 \\
Pressure (atm) $=$ & 5.7 \\
Inlet Gas Temperature (K) $=$ & 1974 \\
Particle Boiling Temperature $(K)=$ & 1594 \\
Inlet Gas Velocity $(\mathrm{m} / \mathrm{s})=$ & 29.3 \\
Inlet Particle Velocity $(\mathrm{m} / \mathrm{s})=$ & 25 \\
Bulk Seed Mass Fraction $(9)=$ & 1 \\
Overall Stoichiometric Ratio $=$ & 1.0 \\
Inlet Fucl Concentration $=$ & 0.368 \\
Inlet Inert Concentration $=$ & 0.390 \\
Inlet Product Concentration $=$ & 0.242 \\
Jet Mass Flow Rate $(\mathrm{kg} / \mathrm{s})=$ & 0.454 \\
Jet Temperature $(\mathrm{K})=$ & 300 \\
Jet Location $(\mathrm{L} / \mathrm{D})=$ & 0.66 \\
Jet Angle (deg.) $=$ & 130 \\
Jet Oxidizer Concentration $=$ & 1.0 \\
\hline
\end{tabular}

Flow Patterns of Non-Vaporizing Particles

For reacting flows laden with single-sized nonvaporizing particles, several cases were computed. Particle flow patterns are found different than those of the gas flow. Particles entering the combustor are pushed either to the combustor center to move around the vortices or to the side walls, via interfacial drag. For very small particles, i.e., particle diameter less than 5 mictons, wall deposition is negligible and most particles flow around the vortices to the combustor center.

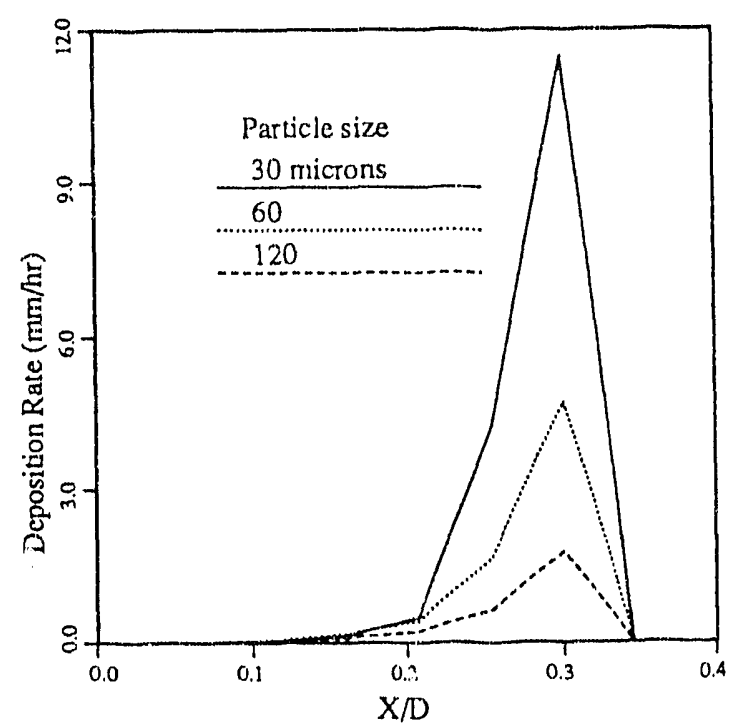

Figure 2. Particle deposition rate on wall for non-vaporizing particles

For larger particles, wall deposition can be significant.
Figure 2 compares particle wall deposition rates for three particle diameters: 30,60 , and 120 mictons. Most significant particle deposition is found near particle flow stagnation points, about half way between the inlet and the jet location. Peak deposition rates at $X / D=0.3$ are 12,5 , and $2 \mathrm{~mm} / \mathrm{hr}$ for 30,60 , and 120 microns cases, respectively under the $1 \%$ particle mass loading condition. Note that at the combustor inlet particles are assumed to flow parallel to the walls. Particle deposition rates downstream of the jets are small because particles are pushed to chamber center as the main flow passes the jets and dispersion back out from combustor center beyond the jets is relatively slow, being primarily a diffusive process.

Figures 3 and 4 show flow patterns of multiple-sized particles in the combustor. This step is essertial before simulating particle evaporation in reacting flows, because particle size changes during evaporation. For simple illustrations, three particle sizes of 20,60 , and 100 microns are chosen and their inlet number densities are assumed to be $2.08 \times 10^{6}, 8.91 \times 10^{7}$, and $8.87 \times 10^{6} \mathrm{\#} / \mathrm{m}^{3}$, respectively.

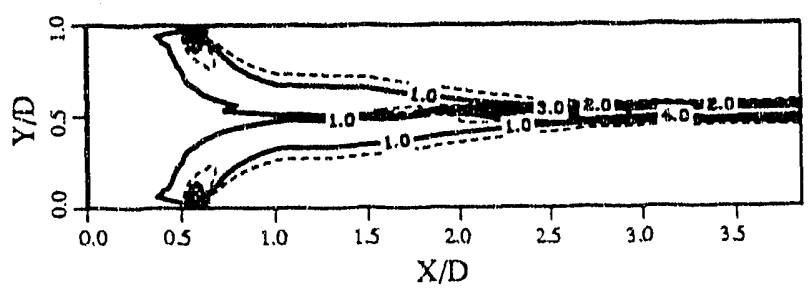

(3a) Number density of total particles

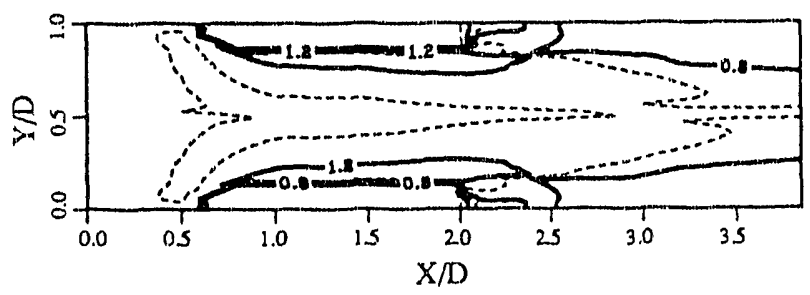

(3b) Average particle size

Figure 3 Dispersion of multiple-size and non-vaporizing particles in the combustor

In Figure 3a, total number density of all particle sizes i: shown. The normalized number density value is $10^{\mathrm{h}} \mathrm{H} / \mathrm{m}^{3}$. Particles are pushed to the center portion of the combustor due to the screening effect of the oxidizer jets. Figure 3b shows local normalized average particle size. The reference particle size used for normalization is the average inlet particle size 34 microns. The variation of local average particle size over the interior of the combustion clearly indicates that particles of 
different sizes have different flow patterns. Figure 4 shows number density plots of each particle size group. Smaller particles follow the main gas flow more closely than larger particles. Thus, smaller particles turn more rapidly toward the center following the main gas flow as it accelerates into the center in order to pass the opposing jet flows. Further, once past the jets, smaller particles begin to spread back out over the chamber cross section more rapidly than larger particles. This more rapid spreading of smaller particles in the downstream is due both to a larger turbulent diffusion coefficient and to a more rapid drag response to the gas flow.

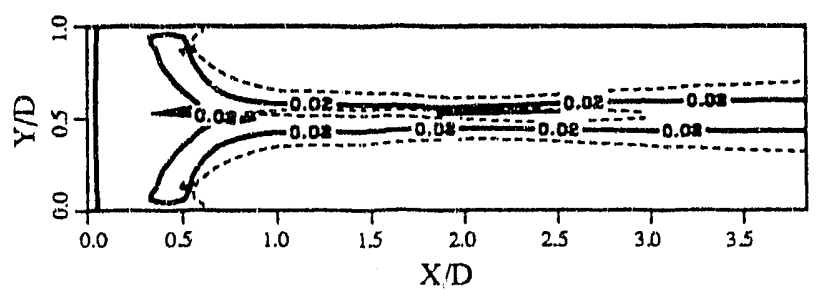

(4a) 20 microns

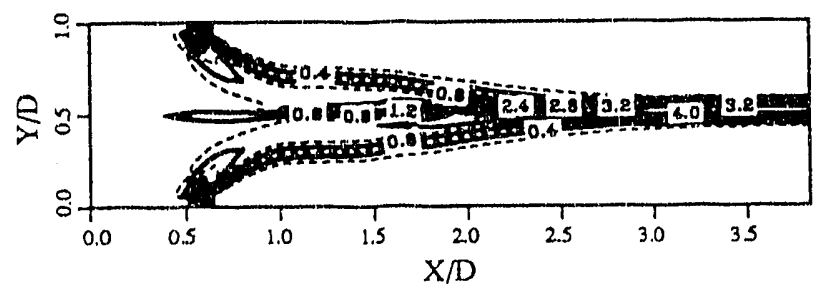

(4b) 60 microns

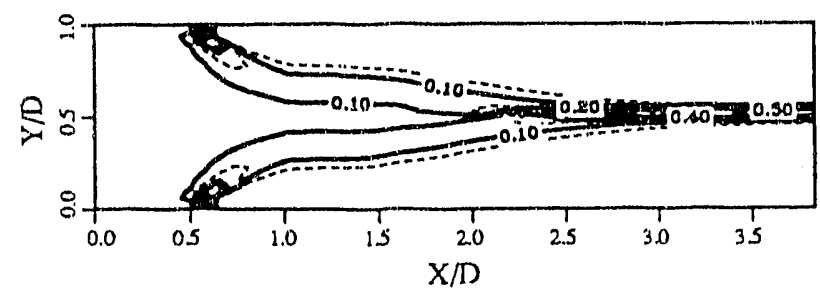

(4c) 120 microns

Figure 4 Particle dispersion of each particle size group as shown in number density contour plots

Flow Patterns of Vaporizing Particles

A baseline case of two-phase combustion flow with vaporizing seed particles is presented in the following paragraphs to give an overview of two phase flow conditions and processes in the combustor and 10 provide a basis for cornparison of the effects of varying mean iniet particle size presented in the next section. The baseline case assumes a uniform inlet particle number density and an average inlet particle size (or diameter) of $34 \mu \mathrm{m}$. Baseline two-phase combustion flow patterns are shown in Figures 5-7. Figures 5a and $5 \mathrm{~b}$ show gas and particle velocity vectors; Figures $6 \mathrm{a}$ and 6b show gas and particle temperature contours; Figure 7a shows total particle number density contours; and Figure $7 \mathrm{~b}$ shows seed vapor concentration contours.

In Figure 5, vector length represents velocity magnitude; inlet gas velocity is $29.6 \mathrm{~m} / \mathrm{s}$; and inlet particle velocity is 25 $\mathrm{m} / \mathrm{s}$. In Figure 6 , temperature is normalized by the reference temperature $2950 \mathrm{~K}$; inlet gas temperature is $1974 \mathrm{~K}$; particle inlet temperature is $300 \mathrm{~K}$; and jet temperature is also $300 \mathrm{~K}$. In Figure $7 \mathrm{a}$, total number density summing number density of five size groups is normalized by its inlet value $528 \times 10^{6} \mathrm{\#} / \mathrm{m}^{3}$. In Figure $7 \mathrm{~b}$, seed vapor concentration is expressed by mass percentage; inlet seed vapor concentration is zero; and inlet seed particle mass flow rate is about $1 \%$ of exit gas mass flow rate.

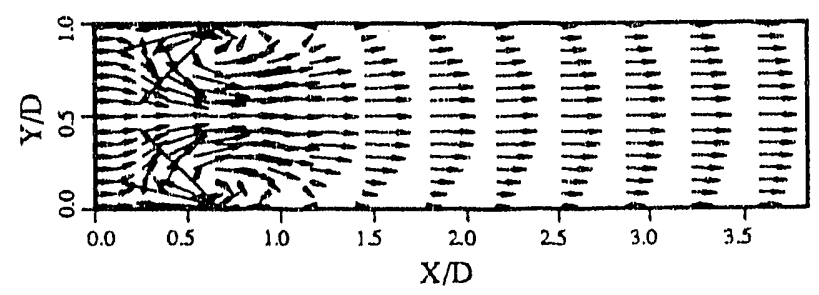

(5a) Gas velocity

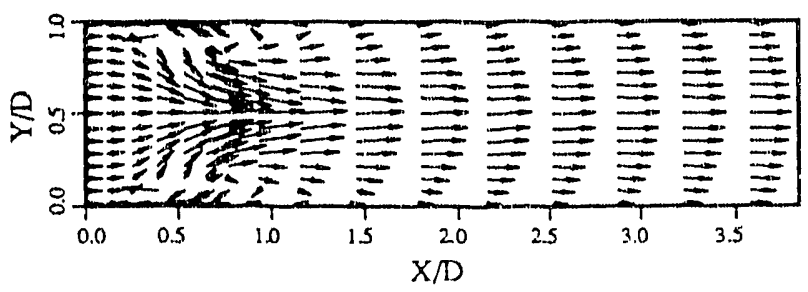

(5b) Particle velocity ( 30 mictons)

Figure 5 Comparison of gas and particle flow patierns 


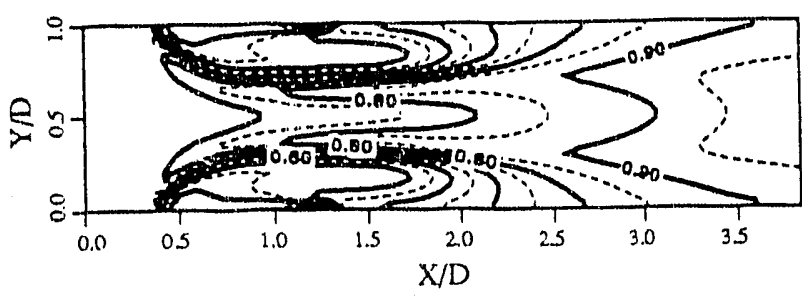

(6a) Gas temperature

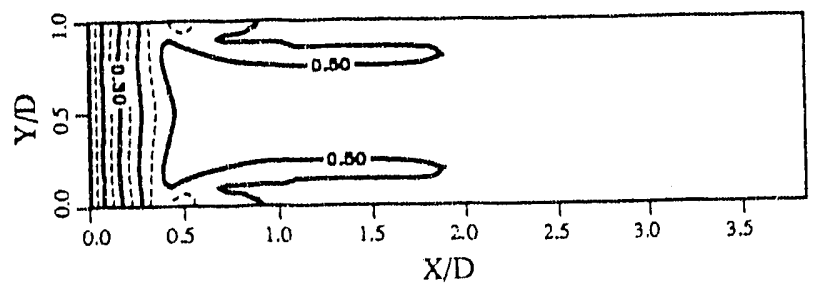

(6b) Particle temperature (30 microns)

Figure 6 Comparison of thermal patterns

With 130 degree counter-flow injection, intense mixing occurs upstream of the injectors at $X / D=0.66$, and the flame is established in this intense mixing zone (Figure 6a). Because the jets penetrate deeply into the main flow, large vortices forn behind the jets (Figure 5a), and the flame follows the vortex boundary from the upstream, where fuel and oxidizer first meet, around toward chamber center as the oxidizer jets are turned downstream. The flame then continues to develop as a diffusion flame in the mixing layer between fuel and oxidizer in the downstrean. Computed results show that the fluid temperature, the fuel concentration, and the oxidizer concentration at the exit are approaching their respective equilibrium values.

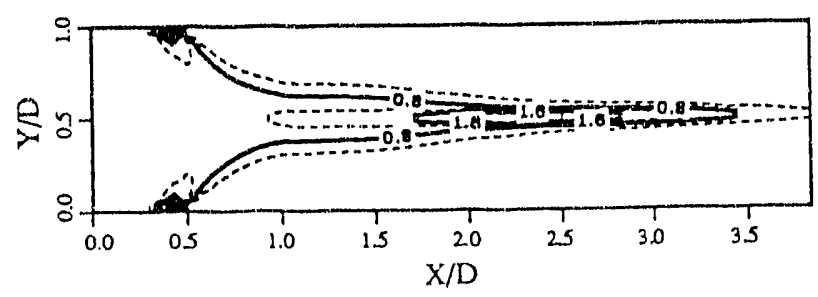

(7a) Particle number density

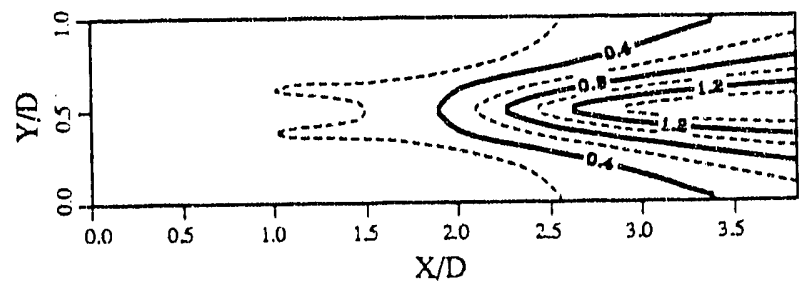

(7b) Seed vapor concentration (\%)

Figure 7 Dispersion of seed particles and vapor

In Figure 5, particle and gas show different flow patterns because of slip velocities. Slip velocity between gas and particles diminishes as the gas flow develops and particles move downstream in the combustor. Because smaller particles react more quickly to the effects of drag, the smaller the particle the smaller the slip velocity. For particles smaller than $5 \mu \mathrm{m}$, slip velocity is negligible. In Figure 6, particle temperature lags gas temperature as particles are heated up by the gas. Slip temperature (or temperature difference) between gas and particles becomes smaller as particles move downstream in the combustor until the particle temperature reaches boiling temperarure. The smaller the particle the faster the particle temperature reaches boiling temperature. For particles smaller than $5 \mu \mathrm{m}$, particle temperature reaches boiling temperature almost immediately. Figure 7a showe that some particles are pushed by the oxidizer jets to the combustor center before they are vaporized; some particles impinge on the wall in upstream of the oxidizer jet openings; and some particles escape the combustor. At builing temperature, $\mathrm{T}_{\mathrm{b}}=$ $1595 \mathrm{~K}$, particles vaporize at a rati depending on the surrounding gas temperature and slip velocity. Figure $7 \mathrm{~b}$ shows that seed vapor is formed primarily near the combustor center and is gradually diffused to the side walls as the gas flows downstream.

The predicted combustion flow patterns for various oxidizer jet angles have been computed and compared. Oxidizer jet angle is found to have a large effect on combustion performance as well as particle cvaporation. Predicted flow pattems of a 50 degree oxidizer jet injection case contrast sharply with those of 130 degree oxidizer jet injection described above. For 50 degree injection the oxidizer jets do not penetrate significantly into the main flow, but rather are rapidly turned into the downstream forming a thick high gradicnt region near the walls. This flow configuration creates a nearly pure diffusion flame with a relatively low rate of mixing and combustion compared to the 130 degree injection case. Clearly, the change of combustion flow patterns affects the combustion performance, especially, the uniformity of temperature profile at the exit plane (or exit thermal 
mixedness), which has great influence on the particle vaporization and vapor dispersion. The dependence of exit seed vapor mixedness on jet angle for a two-phase combustion flow is found similar to those of thermal mixedness for gas combustion flow and fluid mixedness for non-reacting flow. A large increase in particle vaporization occurs when going from co-flow injection to counter-flow injection. The angle range for most effective particle vaporization and vapor dispersion is approximately 130 degrees.

Although there is no experimental data for direct comparison with the two-phase combustion calculations, the prediction of optimum jet angle appears to be in general agreement with preliminary testing results and the predicted optimum jet angle matches the design of TRW's MHD second stage combustor [7].

\section{Effect of Particle Size on : ?d Vapor Dispersion}

Particle size has a significant effect on overall particle evaporation (larget particles take longer to vaporize) with consequent impact on seed vapor mixing and uniformity of the seed vapor profile at the combustor exit. Cornputations were performed for four different inlet mean particle diameters in the range 8.5 to $68 \mu \mathrm{m}$. One set of computations assumed a uniform distribution of particles across the chamber inlet. $A$ second set of computations assumed a normal distribution with the peak of the inlet particle number density occurring at the chamber midplane.

Results presented in the next few paragraphs are for cases of uniform inlet number density distribution. The change of total number density (the sum of number density over the particle size spectrum at a point) along a line of grid points adjacent to the chamber center line and here after referred to as the center line is shown in Figure 8 for various inlet mean particle diameters. The injected particles are assumed to be cold relative to the incoming gas stream from the first stage combustor. Before vaporizing, the particles must be heated by the surrounding gas. This prevaporization heating takes longer for large particles than for small particles, and therefore number densities for cases with larger size particles remain higher into the downstream of the chamber. A second process affecting particle number density at chamber center is the screcning effect of the jets. The main flow is forced toward chamber center in order to pass the sidewall oxidizer iets, and particles are pushed into the chamber center in this process through drag effects. For the smallest particle case (mean diameter $8.5 \mu \mathrm{m}$ ) most of the vaporization takes place upstream of the jets at $(X / D=0.66)$, and therefore, the total center line number density for this case drops rapidly and continuously due to vaporization as the particle flow moves downstream from the inlet. For the $341 \mathrm{~m}$ diameter case, a large buildup of particle number density in chamber center is seen in Figure 8 due to the screening effect of the jets. Larger particles take significantly longer time to heat and to vaporize than smaller particles. As shown in Figure 8 for the $34 \mu \mathrm{m}$ diameter case, center line number density remains above 40 percent of the inlet value all the way to the exit plane. Results show that for the larger inlet mean particle dianeter cases $(34 \mu$ $\mathrm{m}$ and above), significant numbers of particles escape the chambet before vaporization has been completed near the chamber center.

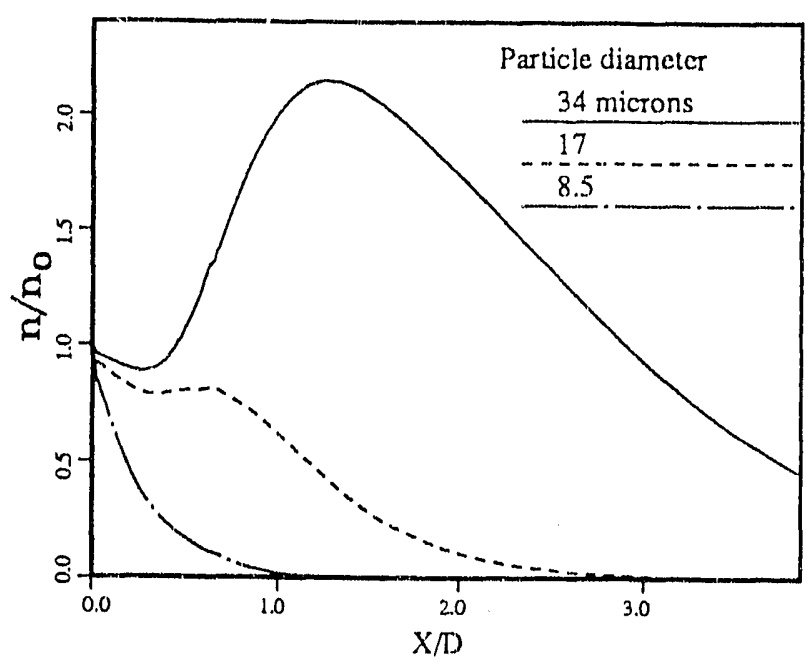

Figure 8 Centerline total number density of a particle laden combustion flow with various mean particle sizes

Of course, number density is not a measure of the mass fraction of the seed particles that has vaporized at the chamber exit plane, because as particles vaporize they dectease in size, but they still exist and contribute to the total number density until they have vaporized completely and their diameter becomes zero. The fraction of injected particle mass vaporized at combustor exit for the $34 \mu \mathrm{m}$ case is about 90 percent for the cases of smaller particles $(17$ and $8.5 \mu \mathrm{m}$ mean inlet diameter) over 98 percent was vaporized by chamber exit, while for the largest mean inlet diameter case tested $(68 \mu \mathrm{m})$, the fraction of inlet particle mass vaporized dropped to about 40 percent.

Both the extent of vaporization and the location of vaporization vary with mean inlet particle size over the range of particle sizes ested. As previously noted particles that have not vaporized upstream of the jets are pushed via interfacial drag to the chamber center. Due to the presence of recirculation zones behind the jets, the spreading of the main now back across the chamber in the downstream of the jet location is gradual. As a consequence, the component of particle drag away from the center line in the downstream of the jets is very small, and particles tend to remain concentrated near the chamber center line in the region downstream of the 
jets. Therefore, when vaporization of larger particles occurs downstream of the jets due to longer prevaporization heating delay and longer vaporization time, seed vapor is primarily deposited near the chamber center in that downstream region. In the MHD application, a uniform distribution of seed vapor is desirable, and delayed vaporization for larget seed particle sizes makes the exit distribution of seed vapor much less uniform. This result is shown is Figure 9 where exit seed vapor concentration profiles are plotted. For the cases with mean inlet particle diameter of 8.5 and $1 \% \mu \mathrm{m}$, the seed vapor distribution at the exit is fairly uniform. For the $8.5 \mu \mathrm{m}$ case much of the vaporization occurs upsteam of the jets. In the 17 $\mu \mathrm{m}$ case a large portion of the vaporization occurs downstream of the jet inlet plane, and therefore near the chamber center, however, most of the vaporization still occurs far enough upstream of the chamber exit for th a vapor deposited near chamber center to spread out across the chamber via diffusion and turbulent transport, giving a relatively uniform seed vapor distribution at the chamber exit plane. For the case of $34 \mathrm{k} \mathrm{m}$ mean inlet particle diameter, much of the vaporization occurs too far into the downstream of the chamber for the spreading effects of diffusion and turbulence to have much impact by the time the flow reaches the chamber exith and therefore the seed vapor distribution for the $34 \mu \mathrm{m}$ inlet mean particle size case has a pronounced peak in the chamber center $(Y / D=0.5)$, Figure 9.

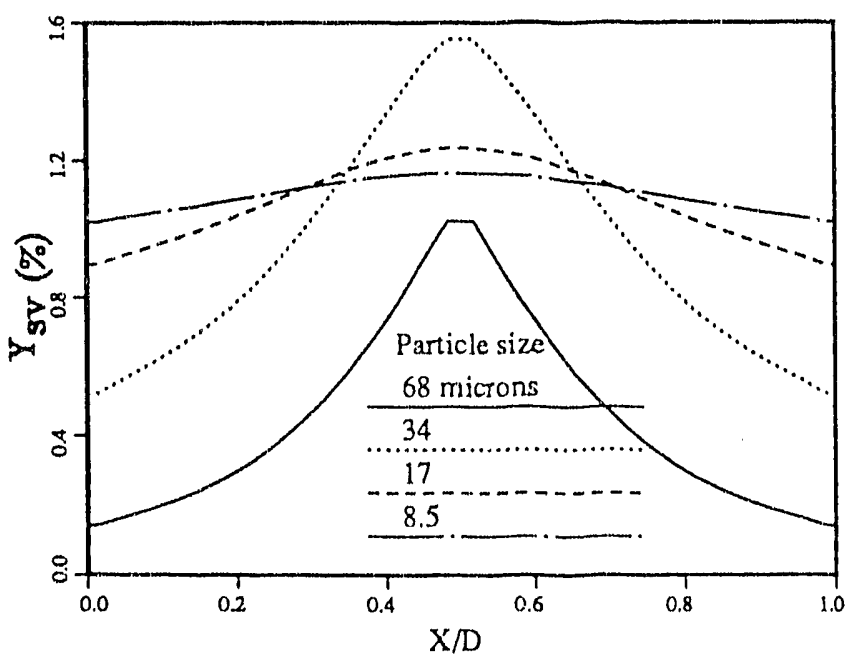

Figure 9 Comparison of exit seed vapor concentration profiles for various mean particle diameters

The presence of vaporizing particles in the flow may affect the combustion progress through heat removed from the gas phase for vaporization, dilution of reactants with seed vapor, slight changes in hydrodynamics due 10 momentum transfer via interfacial drag, and turbulent kinetic energy removed from the gas phase also due to interfacial drag. In the cases tested, which all used a particle loading of approximatcly
1 percent by mass, the effect of inlet mean particle size on combustion progress, measured by the change of center line fuel concentration, was small. The peak gas temperature (near $3000 \mathrm{~K}$ ) drops about $50 \mathrm{~K}$ due to the presence of particles.

A second set of cases was computed with a normal distribution of iniet number density. This distribution concentrates particles in the chamber center at the inlet and simulates an injector source of particles in the near upstream of the inlet plane. As noted in the discussion of cases with a uniform particle distribution at the inlet, the screening effect of the oxidizer jets at $X / D=0.66$ tends to push the particle flow into the chamber center. The difference between a uniform inlet distribution of particles and a normal distribution with number density peak in the center is primarily a consequence of processes upstream of the jets. The main difference in vaporization and seed vapor distribution occurring between the uniform and nermal distribution of paricles at the inlet is an increase in the portion of vaporization occurring near the center line primarily for the cases of smaller iniet mean particle size. Because this increase in near center deposition of seed vapor occurs primarily upstream of the jets, sufficient length of chamber remains for much of this vapor to spread out over the chamber cross section as the flow proceeds downstream. Consequently, seed vapor distributions are slightly but not dramatically less uniform at the chamber exit for the normal inlet distribution cases than for the uniform inlet distribution cases, and the effect is more pronounced for the smaller inlet mean particle sizes.

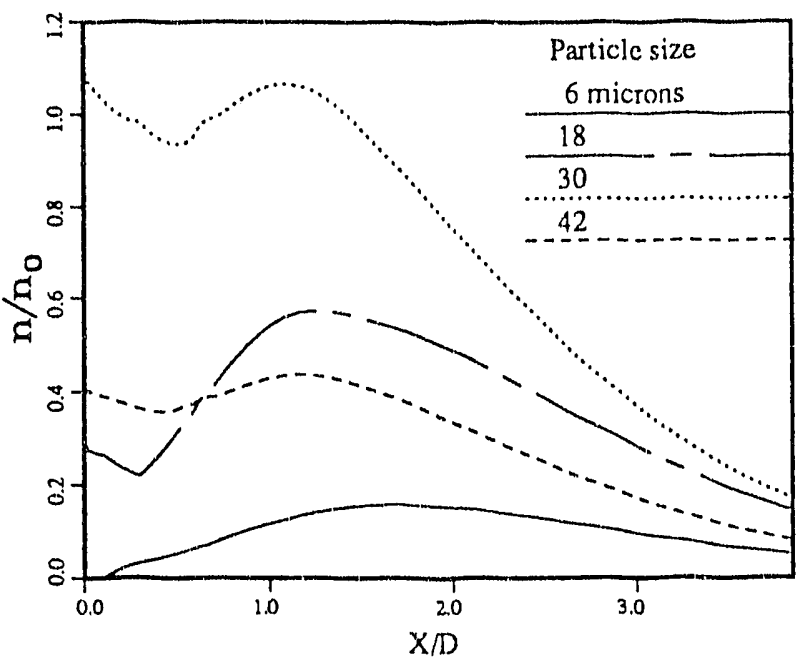

Figure 10 Centerline particle number density of sever individual size groups

The computation of each case also uses a normal distribution of particle sizes about the inlet mean particle size to represent the inlet particle size spectrum. For purposes of discretc computation on a digital computer, the size spectrum is 
divided into a number of particle size bands or groups. The change of particle number density along the center line for the case with an inlet mean particle diameter of $34 \mu \mathrm{m}$ is shown in Figure 10 for several of the individual size groups. The vaporization process in a particular size band decreases the number density in that size group and increases the number density in the next smaller size group. The screening effect of the jets also tends to increase number density in all size groups as particles are pushed to the center.

\section{CONCLUSION}

A comprehensive computer code has been developed for the simulation of a particle laden combustion flow in an MHD second stage combustor. The simulation is used to investigate the effects of combustor operating parameters on seed particle evaporation and vapor dispersion because the cross-sectional uniformity of gas temperature and seed vapor concentration is crucially important to the performance of the down stream MHD power generator. A sensitivity study indicated that a grid of 54 by 32 nodes to represent the combustor configuration and five particle size groups to represent the size distribution for a two-phase combustion flow simulation are adequate to numerically model many of the imporiant characteristics of the combustor operation. Convergence criteria were set to a mass residual of $10^{-9}$

for the gas phase and $10^{.5}$ fortheparticlephase. Particle now pattems are found different than gas flow patterns because of slip velocities and consequent effects of interfacial drag. Slip velocity and slip temperature of smaller particles become negligible. In the combustor, many particles are pushed by the oxidizer jets to the combustor center for vaporization and some fraction of the particles impinge on the walls upstream of the jets. Depending on the particle size, some particles may escape the combustor before they are completely vaporized. Most larger particles are heated to boiling temperature and vaporize in combustor center, then, seed vapor diffuses (via combined turbulent and laminar diffusion) to the side walis. Results also show that the oxidizer jet angle and particle size have are strongly related to particle evaporation and vapor dispersion. At a jet angle about 130 degrees, the overall particle evaporation rate is the highest because of the highest average gas temperature and combustion efficiency. For seed particles having a small mean diameter, particle vaporization in the combustion flow is more complete and vapor dispersion at the exit is more uniform. As particle size increases beyond 10 microns in diameter, the effects of particle size on wall deposition rate, evaportaion delay, and downstream seed vapor dispersion become more pronounced.

\section{ACKNOWLEDGEMENT}

This work was supported by U.S. Department of Energy, Assistant Secretary for Fossil Energy, Under Contract W-31-109-ENG-38, Valuable information provided by TRW, Inc, for this study is appreciated.

\section{REFERENCES}

[1] Chang, S.L., G.F. Berry, and N. Hu, "System Analysis of High Performance MHD Systems," Proc. of 23rd Intersocicty Energy Conversion Engineering Conference, Vol. 4, pp. 455-459 (1988).

[2] Carter C. and J.B. Heywond, "Optimization Studies on Open-Cycle MHD Generators," AIAA Journal, Vol. 6, pp. 1703-1711 (1968).

[3] Chang, S.L., S.A. Lottes, and G.F. Berry, "Computation of Two-Dimensional Non-Reacling Jet/Main Flow Mixing in an MHD Second Stage Combustor," Numerical Heat Transfer, Part A, 20(2):223.236 (1991).

[4] Lottes. S.A., and S.L. Chang, "Simulation of Combustion Processes in an MHD Second Stage Combustor," Proc. of 29 th Symposium of Engineering Aspects of Magnetohydrodynamics, New Orleans, LA, pp.III.4.1-11 (1991)

[5] Berry, G.F., S.L. Chang, S.A. Lottes, and W.A. Rimkus, "Multi-Dimensional Computer Simulation of MHD Combustor Hydrodynamics," ALAA 22nd Fluid Dynamics, Plasma Dynamics \& Lasers Conference, Honolulu, Hawaii, AIAA 91-1511 (June 24-26, 1991)

[6] Lottes, S.A., S.L. Chang, and G.F. Berry, "Effects of Jet Port Arrangement on Three-Dimensional Non-Reacting Jet-Gas Mixing in an MHD Second Stage Combustor," Proc. of 25th Intersociety Energy Conversion Engineering Confetence, Reno, Nevada, 2:468-473 (August 12-18, 1990).

[7] Grove, A., "Design Study of MHD Coral-Fired Combustor Second Stage," 29th Symposium on Enginecring Aspects of Magnetohydrodynamics, New Orleans, LA, pp.III.3.1-III.3.9 (June 18-20, 1991).

[8] Launder, B.E., and D.B. Spalding, "The Numerical Computation of Turbulent Flows," Computer Methods in Applied Mechanics and Enginecring, Yol. 3, pp. 269-289 (1974).

[9] Patankar, S.V., "Numerical Heat Transfer and Fluid Flow." McGraw-Hill Book Company (1980). 
[10] Zhou, X.Q., and H. Chiu, "Spray Group Combustion Processes in Air Breathing Propulsion Combustors," Paper AIAA-83-1323, AlAASAE/ASME 19th Joint Propulsion Conference, Seattle, Washington (1983).

[11] Patankzs, S.V., D.K. Basu, and S.A. Alpay, "Prodiction of the Three-Dimensional Velocity Field of a Deflected Turbulent Jeh." Journal of Fluids Engineeririg. 99:758-76'2 (1977).

[12] Jiang, T.L., and H.H. Chiu, "Advanced Modeling of Spray Combustion Process in Air Breathing Propulsion Combustors," AIAA-87-006\%, MLAA 5th Ac. rnace Science Meeting, Reno, Nevada (1987).

[13] Loues. S.A., and S.L. Chang "Interactions of rurbulent Eddies and Combustion in an MHD Second Stage Combustor," Procecdings of the 26th Intersocicty Energy Conversion Enginerring Conference, Boston, MA, 3:69-74 (August 4-\$.
1991).

[14] Chang, S.L., and́ C.S. Warig, "Thermal Radiation and Spray Group Combustion in Diesel Engines," ASME Winter Annual Meeting, Boston, Mass., HTD-81:25-34 (December 13.18, 1987).

[15] Biuker, D.A. and V.J. Scullin, "General Chemical Kinetics Computer Program for Static and Flow Reactions, with Application to Combustion and Shock-rube Kinetics," NASA TN D.6586 (1972).

[16] Ward, P., N. Collings, and N. Hey, "A Comparison of Simple Models of Turbulent Droplet Diffusion Suitable for Lise in Computations of Spray Flames," ASME 82-WA/HTT-? (1982). 

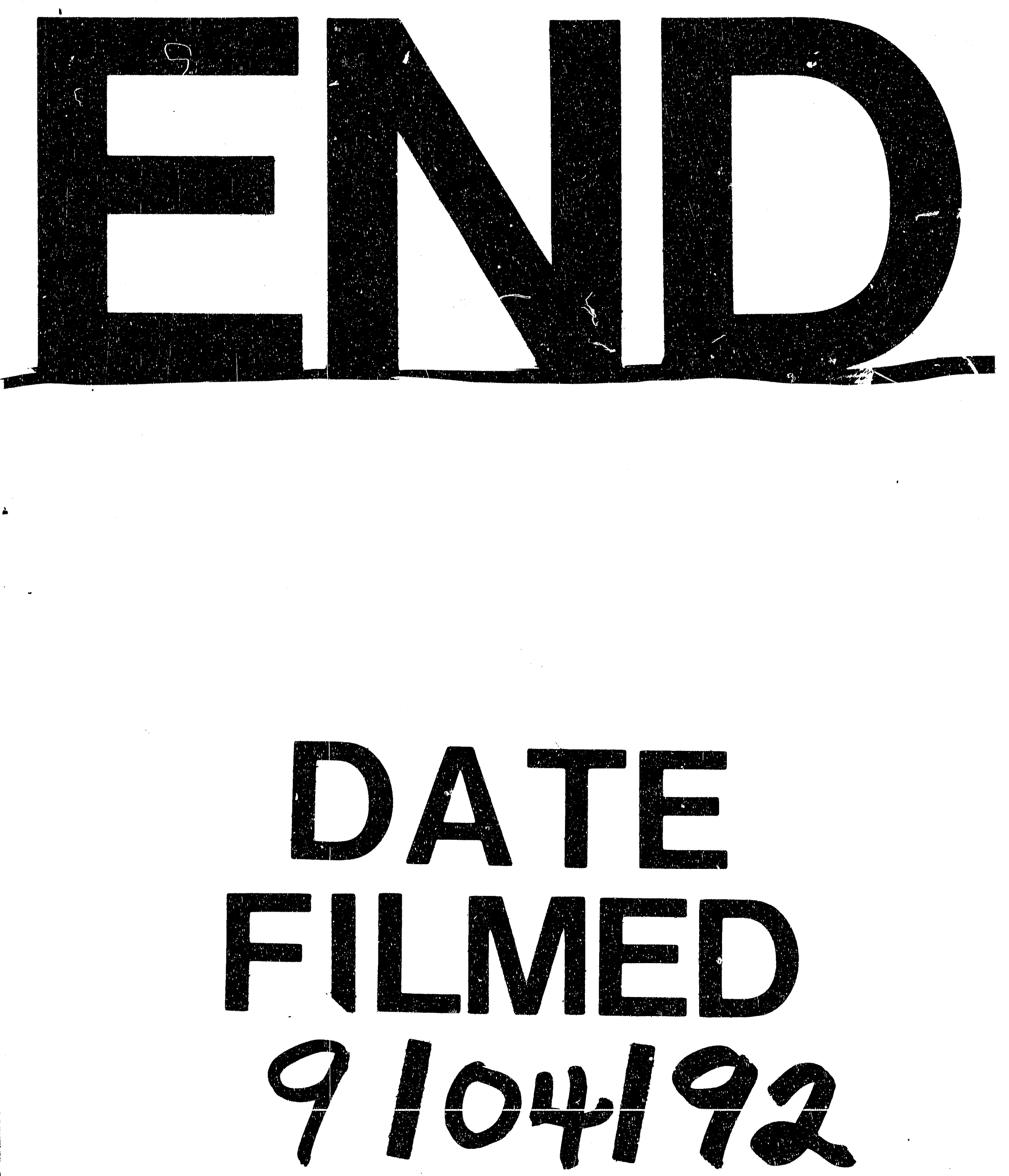
